\title{
Tomographic reconstruction of internal wave patterns in a paraboloid
}

\author{
Jeroen Hazewinkel • Leo R. M. Maas • \\ Stuart B. Dalziel
}

Received: 10 November 2009/Revised: 30 April 2010/Accepted: 1 June 2010/Published online: 6 July 2010

(C) The Author(s) 2010. This article is published with open access at Springerlink.com

\begin{abstract}
Using tomographic synthetic schlieren, we are able to reconstruct the three-dimensional density field of internal waves. In this study, the waves are radiating from an oscillating sphere positioned eccentrically at the surface of a paraboloidal domain filled with a uniformly stratified fluid. We find that the prediction by ray tracing corresponds well with the observed intensities of the wave field. Remarkably, for a specific value of the forcing frequency, we observe convergence of internal wave energy to an internal wave attractor. The attractor is found to dominate fluid motion in the plane perpendicular to the plane spanned by the symmetry axis and the oscillator position.
\end{abstract}

J. Hazewinkel · L. R. M. Maas

Royal Netherlands Institute for Sea Research,

P.O. Box 59, 1790 AB Texel, The Netherlands

J. Hazewinkel · S. B. Dalziel

Department of Applied Mathematics and Theoretical Physics,

University of Cambridge, Wilberforce Road,

Cambridge CB3 0WA, UK

J. Hazewinkel

Centrum Wiskunde \& Informatica, P.O. Box 94079,

1090 GB Amsterdam, The Netherlands

L. R. M. Maas

Institute for Marine and Atmospheric Research Utrecht,

University Utrecht, Princetonplein 5,

3584 CC Utrecht, The Netherlands

J. Hazewinkel $(\bowtie)$

Scripps Institution of Oceanography, University of California, San Diego, 9500 Gilman Drive, La Jolla, CA 92093-0209, USA e-mail: jhazewinkel@ucsd.edu

\section{Introduction}

Stably stratified fluids support internal waves that travel under an angle with respect to gravity (Görtler 1943). This angle is determined solely by the frequency of the forcing and the background stratification (LeBlond and Mysak 1978). In a uniformly stratified fluid, a periodic disturbance causes the wave energy to radiate out in an internal wave cone. In an essentially two-dimensional (2D) setting, this cone is reduced to the well-known St. Andrew's cross (Mowbray and Rarity 1967) that led to the concept of internal wave beams (Thomas and Stevenson 1972; Tabaei and Akylas 2003). In quasi two-dimensional laboratory setups, these internal wave beams were observed as generated by oscillating cylinders or slopes converting surface waves into internal waves (Mowbray and Rarity 1967; Sutherland et al. 1999; Baines 1974; Gostiaux et al. 2006; Echeverri et al. 2009). In the two-dimensional setting, the dispersion relation predicts that upon reflection at sloping walls, focussing or defocussing of the wave energy of these beams will occur (Dauxois and Young 1999; Mercier et al. 2008). In enclosed domains focussing dominates and leads to the formation of internal wave attractors (Maas and Lam 1995). This theory has been verified in experimental studies, again using a quasi 2D laboratory set-up (Maas et al. 1997; Hazewinkel et al. 2008, 2010a).

All these laboratory experiments were in reality threedimensional (3D), but domain and source were chosen such that the waves were essentially uniform in the transverse direction. Most studies employed schlieren methods and PIV (Particle Image Velocimetry), assuming the 2D structure valid up to first order (Hazewinkel et al. 2010a). One of the canonical papers on internal waves (Mowbray and Rarity 1967) was in fact observing the internal wave cone from an oscillating stick but the schlieren method 
used could not resolve this fully three-dimensional wave field. The observations seem to be in a plane. Up till now, only preliminary experimental work on the $3 \mathrm{D}$ observation of axisymmetric internal wave structures from oscillating and falling spheres has been presented (Onu et al. 2003; Yick et al. 2006), but no rigorous experiments have been performed on the full 3D internal wave field structure.

Meanwhile, from the theoretical and numerical side, internal wave focussing has been considered in three dimensions (Maas 2005; Bühler and Muller 2007; Drijfhout and Maas 2008). The full three-dimensional structure of the internal wave field may lead to patterns that are not axisymmetric. Such patterns cannot be observed by standard schlieren techniques but can be reconstructed using tomography. Predicting the patterns set by a simple disturbance in a three-dimensional enclosed domain is a mathematical difficulty and wave fields have so far only been approached by ray tracing from a point source (Maas 2005). For some specific parameters, this ray tracing reveals the formation of internal wave attractors, i.e. limit cycles where all rays converge. However, as ray tracing does not take phase and interference processes into account, it remains uncertain if 3D domains allow for attractors.

Although the full tomographic reconstruction of internal wave patterns in an enclosed stratified fluid has not been considered before, the general problem of tomographic reconstruction of density distributions from deflection and schlieren data has been studied before. The 3D density distributions have been reconstructed for a supersonic jet (Faris and Byer 1988), the temperature field in gas-flows (Agrawal et al. 1998) and the concentration field around a growing crystal (Srivastava et al. 2005). In all these experiments, the reconstruction is based on beam deflection by a density perturbation, the principle also underlying

Table 1 Parameters of experiment

\begin{tabular}{lll}
\hline Parabolic tank & & \\
Height & $R$ & $300 \mathrm{~mm}$ \\
Radius & $L$ & $300 \mathrm{~mm}$ \\
$\quad$ Distance camera & & $8,500 \mathrm{~mm}$ \\
Fluid surface & $H$ & $290 \mathrm{~mm}$ \\
Height & & $295 \mathrm{~mm}$ \\
Radius & $N$ & $2.2 \mathrm{rad} \cdot \mathrm{s}^{-1}$ \\
Buoyancy frequency & & \\
Forcing & & $40 \mathrm{~mm}$ \\
Radius sphere & & $0.55 \mathrm{R}$ \\
Radial position sphere & & $10 \mathrm{~mm}$ \\
Amplitude vertical oscillation & $T$ & $5.3 \mathrm{~s}$ \\
Exp. 1: period & $T_{\text {attractor }}$ & $4.3 \mathrm{~s}$ \\
Exp. 2: period &
\end{tabular}

synthetic schlieren (Dalziel et al. 2000) (sometimes known as Background Oriented Schlieren (BOS) (Richard and Raffel 2001)). In this paper, we present the use of tomographic synthetic schlieren (Goldhahn and Seume 2007) to reconstruct the $3 \mathrm{D}$ internal wave patterns from an oscillating sphere positioned eccentrically in a paraboloidal domain with a linearly stratified fluid.

\section{Experimental set-up}

\subsection{Synthetic schlieren}

For the experiments, we use a paraboloidal tank of depth 300 $\mathrm{mm}$ and radius $r=300 \mathrm{~mm}$ at the top (see Table 1 and Fig. 1). The tank is filled with a fluid which has a linear density gradient $\rho_{0}(z)$ in the vertical $z$ to fluid height $H$. The stratification is characterized by buoyancy frequency $N=\sqrt{-g \rho_{*}^{-1} \mathrm{~d} \rho_{0} / \mathrm{d} z}$, with $g$ the acceleration due to gravity and $\rho_{*}$ the constant reference density of water. This is achieved with the use of two, computer controlled, Masterflex peristaltic pumps. The paraboloidal tank is mounted in a larger square tank which is filled with water to compensate for the distortions of the parabolic shaped tank. The distortions of the parabolic tank with the stratification are most dramatic at the bottom, where also the stratified fluid has its highest density. Optically, the optimal situation would be to have the same density stratification in both tanks; however, this would potentially lead to internal wave patterns in both tanks, a far from optimal situation. For these reasons, we fill the outer tank with fluid having the highest density of the stratification in the paraboloidal tank. In this way, we can observe a random dot pattern behind the two tanks on a light bank. This enables us to use synthetic schlieren (Dalziel et al. 2000) to measure the internal wave motion in inner (paraboloidal) tank. Synthetic schlieren relies on the apparent distortion of this background dot pattern due to refractive index changes resulting from density perturbations $\rho$ when looking through the tank.

The use of synthetic schlieren for a three-dimensional density perturbation needs extra attention. Previously, Scase and Dalziel (2006) studied full three-dimensional internal wave patterns in an integrative manner. Although they were not able to resolve the three-dimensional structure of the field, they were able to compare observation with a breadth-averaged (along the line of sight) result from a three-dimensional theoretical prediction. We follow their approach to find the dot-displacement vector $\boldsymbol{\Gamma}$ by tracing light rays through a medium with refractive index $n(x, y, z)$ over width $W$ (the fluid) and distance $B$ to the dot pattern. For simplicity in this discussion we shall ignore the refractive index interfaces between media, although note that necessary corrections (Dalziel et al. 2007) are included 


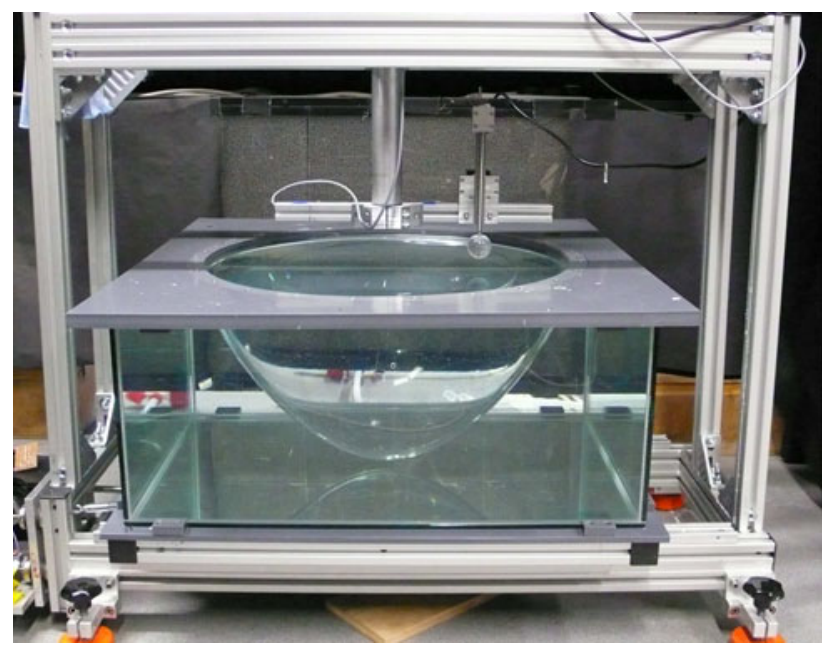

Fig. 1 Photograph of the experimental set-up. The paraboloidal tank is visible in the center of the larger rectangular outer tank. The oscillating sphere, that can be lowered into the fluid, is located above the tank to the right of the center, and the dot pattern on the light bank is behind the tank

in the computation employed for the results. The linearized optics, assuming small deflections to the path of light, give

$\frac{d^{2} \boldsymbol{\Gamma}}{\mathrm{d} x^{2}}=\frac{\nabla_{(y, z)} n}{n_{*}}$,

where $\nabla_{(y, z)}=\left(\frac{\partial}{\partial y}, \frac{\partial}{\partial z}\right)$. The coordinates are the optical axis of the camera $x$, horizontal $y$ and the vertical $z$. The origin of the coordinates is in the center of the tank and $n_{*}$ is the reference value of the refractive index. The displacements are found by integration by parts (see Appendix 1 for details),

$\boldsymbol{\Gamma}=\frac{\nabla_{(y, z)}}{n_{*}}\left[\left(B+\frac{1}{2} W\right) W\langle n\rangle+\int_{-W / 2}^{W / 2} x n \mathrm{~d} x\right]$,

where $\langle n\rangle=W^{-1} \int_{-W / 2}^{W / 2} n \mathrm{~d} x$, the average refraction through the tank. This equation shows that the sensitivity of synthetic schlieren is increased by increasing the distance to the dot pattern (B) (Dalziel et al. 2000). For essentially two-dimensional perturbations of thin experiments $(B \gg W), \int_{-W / 2}^{W / 2} x n \mathrm{~d} x$ is negligible. For a not-so-thin experiment, the sensitivity of the observations will depend on the location of the perturbations in the tank, the closer to the camera, the more sensitive. However, the 'thin experiment' approximation remains valid for any $B, W$ whenever $n$ is symmetric about $x=0$.

To a good approximation, there is a linear relation between the refractive index and the density $\rho$, such that

$\nabla_{(y, z)} n=\beta \frac{n_{*}}{\rho_{*}} \nabla_{(y, z)} \rho$,

with $\beta \approx 0.184$ (Weast 1981). This means that by measuring the apparent displacement vector $\boldsymbol{\Gamma}$ we can find the changes in the perturbation density gradient field
$\boldsymbol{\Gamma}=\beta \frac{\nabla_{(y, z)}}{\rho_{*}}\left[\left(B+\frac{1}{2} W\right) W\langle\rho\rangle+\int_{-W / 2}^{W / 2} x \rho \mathrm{d} x\right]$.

Under the 'thin experiment' approximation, the displacements are obtained as gradient components of the density field, $\nabla_{(y, z)}\langle\rho\rangle$. However, the tomographic reconstruction will be presented in terms of the buoyancy perturbation fields

$\langle b(y, z)\rangle=\frac{g}{\rho_{*}} \nabla_{(y, z)}^{-1}\left(\left\langle\rho-\rho_{0}\right\rangle_{y},\left\langle\rho-\rho_{0}\right\rangle_{z}\right)$,

after integration of the gradient fields (Hazewinkel et al. 2010a).

To record the apparent displacement of the dots, we use a Jai CV-M4+CL camera (1.3 MPixel monochrome). In order to minimize the parallax, the camera is positioned at distance $L$ from the tank and is protected from disturbances in the air by a tent covering most of this optical path. Using an unperturbed reference image, the perturbed position of the dots is translated into corresponding density changes. For this comparison and data processing the DigiFlow software (Dalziel Research Partners, Cambridge) is used.

\subsection{Tomographic reconstruction}

In this section the general principles of tomography are explained; see the Appendix 2 for details. For the tomographic reconstruction of the 3D internal wave field, we need to observe the experiment from many different view angles. Here we will restrict ourselves to angles of rotation $\phi$ about the vertical axis. For any view angle the ray deflection is measured parallel to the $x^{\prime}$-axis, the line of sight. This indicates the introduction of the orthogonal coordinates $x^{\prime}=x \cos \phi+y \sin \phi, y^{\prime}=-x \sin \phi+y \cos \phi$, rotated at view angle $\phi$ from the original coordinates in the $x, y$ plane. The original $y, z$ plane at $\phi=0^{\circ}$ is the plane spanned by symmetry axis and the oscillator position. The synthetic schlieren observations lead to projections $<b(y, z)>$ of the fully three-dimensional $b(x, y, z)$ field, per view angle $\phi$. We will denote these projections as

$$
\begin{aligned}
p\left(y^{\prime}, z, \phi\right)= & \int_{-\infty}^{\infty} b\left(x^{\prime} \cos \phi-y^{\prime} \sin \phi, x^{\prime} \sin \phi\right. \\
& \left.+y^{\prime} \cos \phi, z\right) \mathrm{d} x^{\prime},
\end{aligned}
$$

where $b(x, y, z)=0$ outside the paraboloidal tank. The reconstruction will be by means of the inverse Radon transformation or convolution-backprojection (CBP) algorithm (Faris and Byer 1988; Kak and Slaney 1988), see Appendix 2 for details. At every level $z$ the reconstruction becomes 
$b(x, y)=\int_{0}^{2 \pi}[p(\eta, \phi) * k(\eta)]_{-x \sin \phi+y \cos \phi} \mathrm{d} \phi$,

with $\quad k(\eta)=\int_{0}^{\infty} Y^{\prime} e^{-2 \pi l Y^{\prime} \eta} \mathrm{d} Y^{\prime} \quad$ and convolution $[a(\eta) * b(\eta)]_{\tau}=\int_{-\infty}^{\infty} a(\eta) b(\eta-\tau) \mathrm{d} \eta$. The transformation has become standard in all sorts of tomography and is implemented in Matlab in the image analysis toolbox as the function iradon.m. The function offers a routine to invert the projections, and provides simple ways of applying filters.

\subsection{Forcing of the waves}

Using the symmetry of the paraboloidal tank, the requirement of many view angles is identical to observing from the same angle but rotating the oscillating sphere under an angle $\phi$ about the axis of the tank. This latter, more convenient arrangement is achieved by installing a hub, holding an arm, above the center of the tank. The arm can be rotated about the hub. The vertical oscillator mounted on the arm, drives the sphere of diameter $a=40 \mathrm{~mm}$ at a surface position $r=0.55 \mathrm{R}$. When at rest, the sphere is above the fluid at viewangle $\phi$. Before the oscillation, the sphere is lowered into the fluid, such that it is just below the surface; after the oscillation it is brought back to its original position above the fluid. For the tomographic reconstruction, views on the same plane, i.e. view angles $\phi$ and $\phi+180^{\circ}$, are in theory identical. This means that only the view angles between $0^{\circ}$ and $180^{\circ}$ with a certain interval are needed to reconstruct the internal wave pattern. However, we anticipate a small effect of $\int_{-W / 2}^{W / 2} x \rho \mathrm{d} x$ and perform experiments with the source on both sides of $x=0$. We view angles in regions $\left[0^{\circ}, 180^{\circ}\right)$ and $\left[180^{\circ}+\delta \phi / 2,360^{\circ}+\delta \phi / 2\right)$ with a $\delta \phi$ degrees interval. Under the 'thin experiment' approximation, this is identical to a $\delta \phi / 2$ resolved spatial pattern in a $0^{\circ}-180^{\circ}$ region.

A typical experiment is as follows. In a given stratification, we take a reference image before the sphere is lowered into the fluid, just below the surface. This is followed by oscillating the sphere at a given off-centered position with radius $r$ and given angle $\phi=0$ for 30 periods $T$ at frequency $\omega \equiv 2 \pi / T$, capturing 32 frames per oscillation. When finished, the sphere is brought out of the fluid again and the angle is increased by $\phi+92$. Then, the system is allowed to settle for 2 minutes, typically twice the time needed for the internal waves to dissipate in the fluid. After this pause, the process is repeated from taking the reference onward. The following view angles are $\phi+182$ and $\phi+270$, after which the arm is returned to the initial $\phi$ and the whole loop is repeated for $\phi=\phi+\delta \phi$, until the views are overlapping. In the present paper $\delta \phi=4^{\circ}$ giving 90 views of the experiment.

\section{Results}

We present data from an experiment with forcing frequency $\omega$. Although set-up and typical modus operandi are explained in the previous sections, we will highlight some of the crucial steps in this (time consuming) routine. Running the experiment for all angles with interval $\delta \phi=4^{\circ}$ takes about $11 \mathrm{~h}$. We verify that the stratification remains constant throughout the experiment. Only at the surface, a shallow mixed layer is found, but its maximum depth is less then $15 \mathrm{~mm}$. We analyze the last three periods of the oscillation of all separate experiments and perform harmonic analysis (HA) at the forcing frequency. This results in a complex projection $p\left(y^{\prime}, z\right)$ of the density field for every angle $\phi$, of which the real part is shown in Fig. 2 for four values of $\phi$ (for a better overview of the projections, more values of $\phi$ are shown in Fig. 8 in Appendix 3). The common picture arises with a source from where the internal waves radiate under the typical angle $\theta$, a half version of the familiar 2D St. Andrew's cross (Görtler 1943; Mowbray and Rarity 1967). From all view angles, the beams reflect from the walls and weaken in amplitude further away from the source. The patterns seen are different per view angle $\phi$, but all feature the reflection into the surface corners where the waves weaken due to defocussing. Notice that although the data from different angles reveal different patterns, the waves always have the same angle with the vertical in the synthetic schlieren data.

Using these projections $p\left(y^{\prime}, z\right)$, we perform the tomographic reconstruction for every level $z$ following Eq. 7. As an example, we show the reconstruction of $b(x, y, z=H / 2)$. First, we obtain all projections $p\left(y^{\prime}, \phi\right)$, Fig. $3 \mathrm{a}$, and reconstruct $b(x, y, z=H / 2)$, Fig. $3 \mathrm{~b}$. Clearly seen in the reconstruction is part of an internal wave ring on the left. Note that this example presents only the reconstruction of the real part of the complex projections. The reconstruction is naturally done for both real and imaginary part and combined again to form the complex $b(x, y, z=H / 2)$. This means that also temporal (phase) information can be found from the reconstruction.

The next step in the analysis is then to build the full three-dimensional field using the highest angular resolution. The spatial patterns in the paraboloidal tank are most easily appreciated when plotting some slices of the real component of the reconstruction, Fig. 4. It is seen that the internal wave ring (actually, the cone), grows wider and wider in the downward direction, and that the reflections from the walls distort the circular shape. To understand this picture, we add rays coming from a point source at a location approximately at the position of the sphere in the experiment, Fig. 4b. We plot the first five reflections, and note that the rays and reconstruction coincide closely, at least globally. Local convergence of rays leads to a higher 
Fig. 2 Selection of projections $p\left(y^{\prime}(\phi), z\right)$ after harmonic analysis at the forcing frequency of experiment I, more projections are shown in Fig. 8 in Appendix 3. The contour lines are the values defined in grey scale

Fig. 3 a Projection $p\left(y^{\prime}(\phi)\right.$, $z=H / 2)$ and $\mathbf{b}$ reconstruction of $b(x, y, z=H / 2)$ with angular resolution $4^{\circ}$ for the data of experiment I
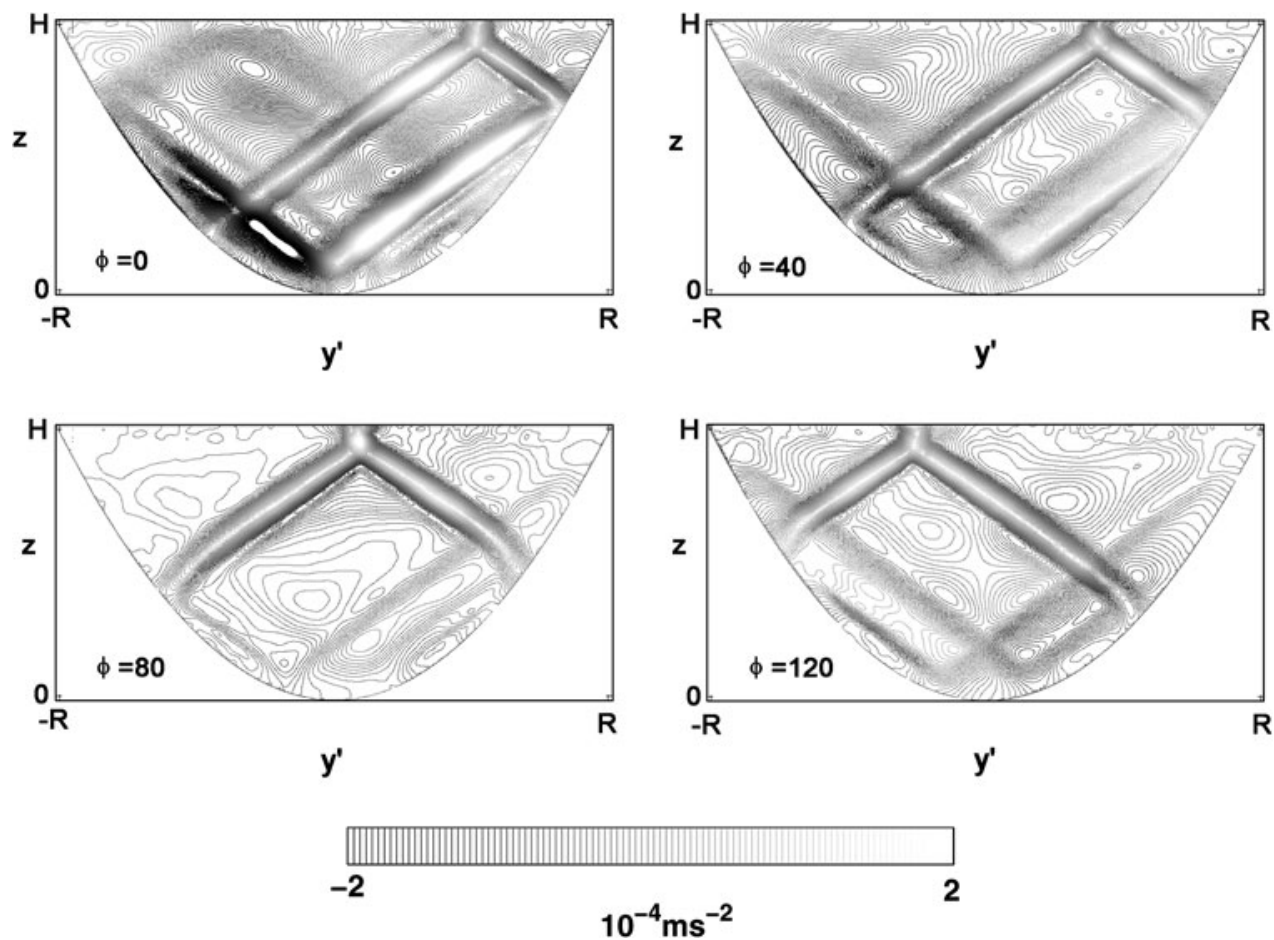

(a)

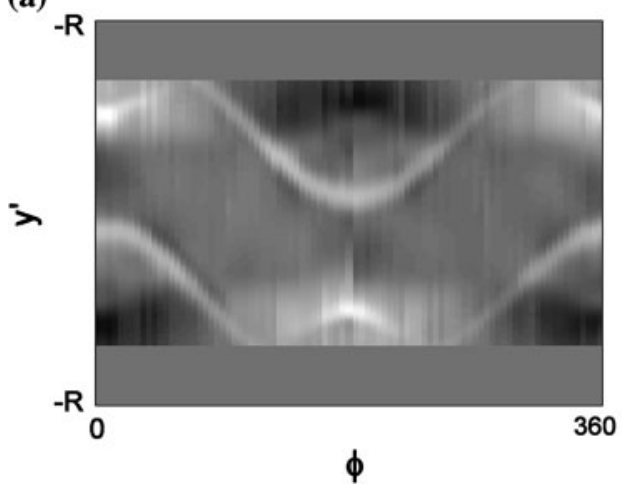

(b)

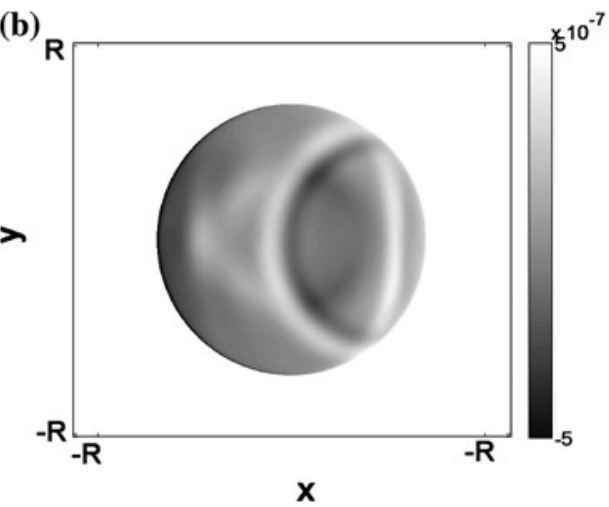

response of the fluid seen in the amplitude plot, and the rays connect regions of equal phase, Fig. 4c,d.

Here we have elected to reconstruct the 3D buoyancy field $b(x, y, z)$. Until recently, most synthetic schlieren data were presented in terms of the vertical component of the gradient field, as this comes naturally from the observations (Hazewinkel et al. 2010a). However, we feel it is more desirable to work with the buoyancy field obtained upon integration of $b_{y^{\prime}}$ and $b_{z}$. We tried the reconstruction of $b_{y^{\prime}}(x, y, z)$ and $b_{z}(x, y, z)$ but found that due to the symmetries in the observations, the reconstruction of $b_{y^{\prime}}(x, y, z)$ is not possible. Since our oscillation is in the vertical, the wave beams close to the source in the observed $b_{y^{\prime}}\left(y^{\prime}, z\right)$ are not mirror-symmetric along the vertical through the source. This means an ideal and naïve reconstruction is zero. However, reconstruction of $b_{z}(x, y, z)$, related to the perturbation in $N$, is possible. In cases where the interest of the data is in the fine structure of the internal wave field this may be the preferred field, due to the highlighting of fine scales as result of the derivative.

\section{Observations of a 3D attractor}

By following the first few reflections of some rays, we obtain a fair approximation of the wave structure. Following the rays for some more reflections leads, for some frequencies, to attractors (Maas 2005). We select such a frequency, $\omega_{\text {attractor, for experiment } 2 \text { and leave all }}$ other settings as in the previous experiment. Again, upon inspection of the snapshots of the observations after harmonic analysis, Fig. 5, we find the half St. Andrews cross (again, an overview showing more projections in found in Appendix 3). Compared to Experiment I, the main 
Fig. 4 Sliceplot of $b(x, y, z)$ in Experiment I. a Real part of HA, b real part of HA including rays from point at surface for 5 reflections, c amplitude and d phase from HA
Fig. 5 Same as 2 but now for experiment II, more projections are shown in Fig. 9 in Appendix 3 (a)

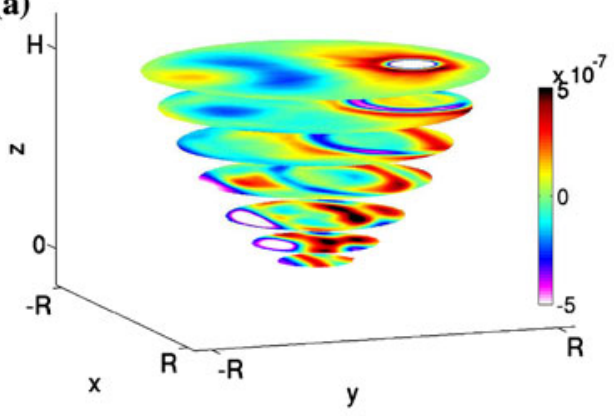

(c)

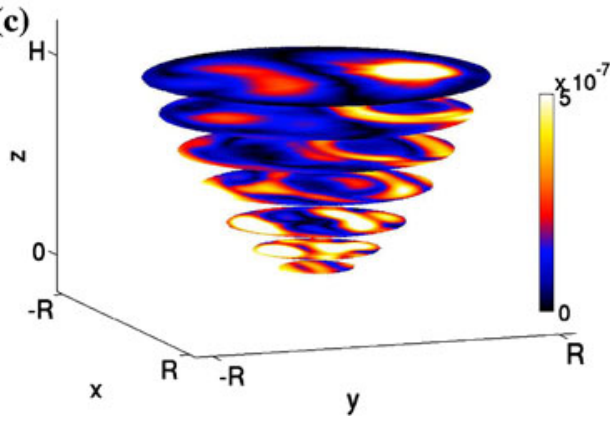

(d)
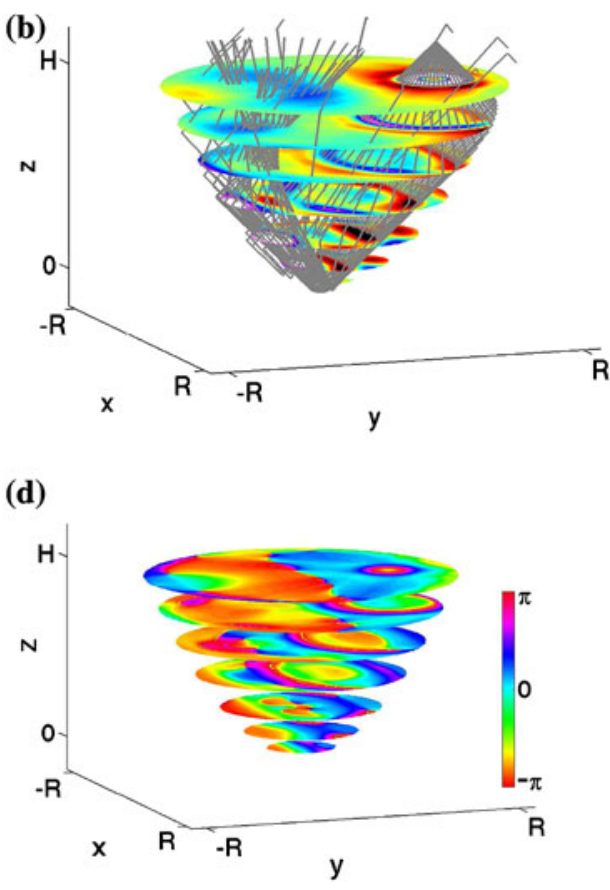
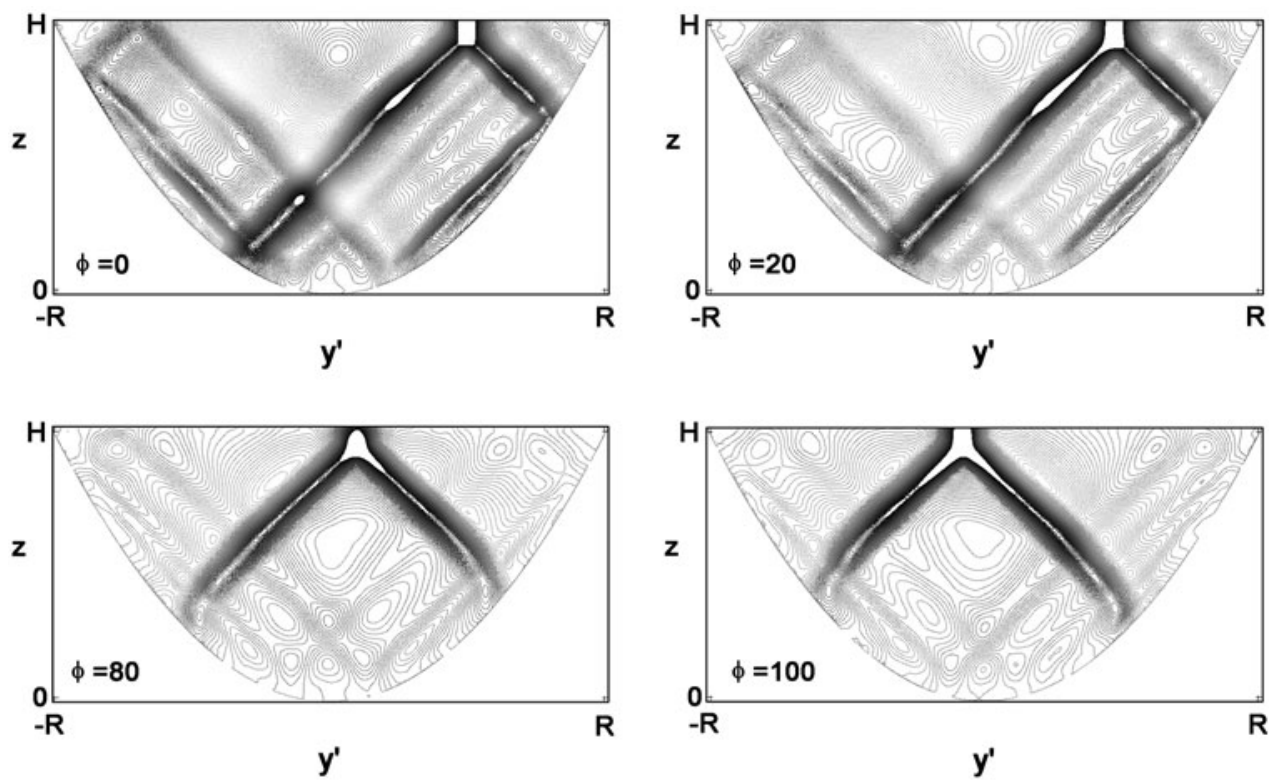

difference is that there seems to be a background pattern, persistent in all views, corresponding with the attractor path we find from ray tracing and experiments in a parabolic channel (Hazewinkel et al. 2010b). This pattern is particularly visible in the all projections of the planes perpendicular to the source, $\phi=80^{\circ}-100^{\circ}$, and in the planes aligning with the source, $\phi=0^{\circ}-20^{\circ}$ (see four of these shown in Fig. 5). We then proceed, as in the previous experiment, to reconstruct the 3D density disturbance and compare it with ray tracing from a cone at the surface.

In the $3 \mathrm{D}$ reconstruction, Fig. 6a, the main signal shows the structure as found from the first 6 reflections (gray rays) 


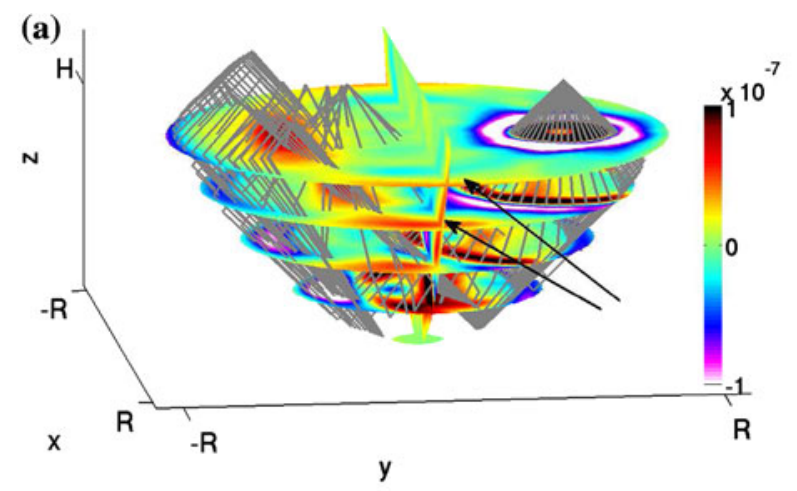

(b)

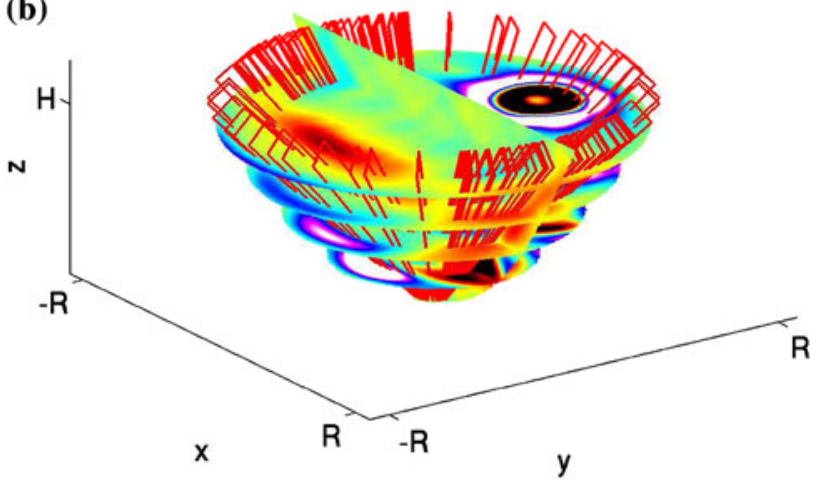

Fig. 6 Comparison of reconstructed buoyancy perturbation in experiment II with ray tracing showing a the first six reflections (gray rays) and $\mathbf{b}$ after 30-50 reflections (red rays). The arrows in (a) indicate motion associated with the attractors found in (b)

of the incoming internal wave cone. Again the internal wave cone is deformed by reflection from the walls leading to energy focussing near the wall opposite to the oscillating sphere. The region of focussing of the rays corresponds with the region of strong internal wave motion, the black and white region in Fig. 6a. There remain some regions, however, where there is a dominant signal that has no ray convergence in the first reflection, indicated by the two arrows. These regions correspond to the region where attractors are found after about 30 reflections of the rays, indicated by the red rays indicating reflections $30-50$ in Fig. 6b. Note that the ray tracing leads to a convergence of rays on attractors near the vertical plane perpendicular to the vertical plane of the forcing and the middle of the tank.

\section{Sensitivity of results}

The reconstructions in previous sections were done with rather high angular resolution, $\delta \phi=4^{\circ}$. To investigate the sensitivity of the reconstruction to the angular resolution $\delta \phi$, the reconstruction is also performed with a subset of the projections. The reconstructions for $\delta \phi=12^{\circ}, 24^{\circ}$ show that, with relatively limited views, a rough idea of the field can still be reconstructed, though the reconstruction loses sensitivity as the views overlap less, Fig. 7b,c. Comparing several reconstructions at a circle with radius ${ }_{9}^{4} R$ with increasing $\delta \phi$ (Fig. 7d), we see that the reconstruction can not be trusted anymore for $\delta \phi \geq 36^{\circ}$. From this resolution onwards, the reconstruction gives rise to spurious patterns at the magnitude of the signal. Although the above reconstructions were done after the harmonic analysis of the data, this step is not necessary. A snapshot that is underlying the reconstruction of Fig. 7a is shown in $7 \mathrm{e}$, showing very a very similar pattern at at a different phase. This allows the analysis of non-periodic events, such as the initial transients, to be reconstructed.

In order to check if the 'thin experiment' approximation is still valid for our not-so-thin experiment, we could compare observations with the source in front and back $\left(\phi=90^{\circ}\right.$ and $\phi=270^{\circ}$ ) of the tank, as for a 'thin experiment' these would be identical. However, we did not capture these overlapping views (see Sect. 2.3). Instead, we choose to perform the reconstruction again, exploiting the symmetry of our experiment in a different way. Knowing the symmetry of our wavefield about $x=0$ we perform a new reconstruction by simply converting all $\phi>180^{\circ} \rightarrow \phi^{\prime}=\phi-180^{\circ}$. We compare this 'thin experiment' reconstruction with the reconstruction as presented in previous sections, along the $x$-axis, $b(x, 0, H / 2)$ see Fig. $7 \mathrm{f}$. The solid line gives the reconstruction as in previous sections, the dotted line gives the reconstruction under the thin experiment assumption. As expected, the original reconstruction had a different value of $b$ in front and back of the tank, due to the sensitivity change. This difference is largely removed in the new reconstruction. Both reconstructions are equal along $y$. However, the differences are small and do not change the qualitative shape.

Using the symmetry once more, we can also average observations from $\phi$ and $180^{\circ}-\phi$, by horizontally flipping one of the two. This means we can remove any error related to the tank not being perfectly normal with the line of sight. In doing so, we find that this 'centered' reconstruction is not very different from the thin experiment reconstruction. This indicates the alignment of the camera is not of major importance for the reconstruction, though it may be optimized by ensuring that the middle of the tank is in the middle of the view.

\section{Discussion and conclusion}

The three-dimensional internal wave field from a spherical oscillator in a paraboloidal domain was successfully reconstructed using tomographic synthetic schlieren. However, the data were not captured instantaneously but rather in a succession of separate experiments exploiting the symmetries of the domain. This could be done as the 
Fig. 7 Reconstruction of $b(x, y, z=H / 2)$ in experiment I with angular resolution a $4^{\circ}$, b $12^{\circ}$ and c $24^{\circ}$. d Reconstructions at different angular resolution along a circle with radius $\frac{4}{9} R$. e Snapshot of the recontruction. f Reconstruction $b(x, y=0, z=H / 2)$ as in previous sections (solid line), for the thin experiment (dashdot line) and the centered experiment (dotted line)
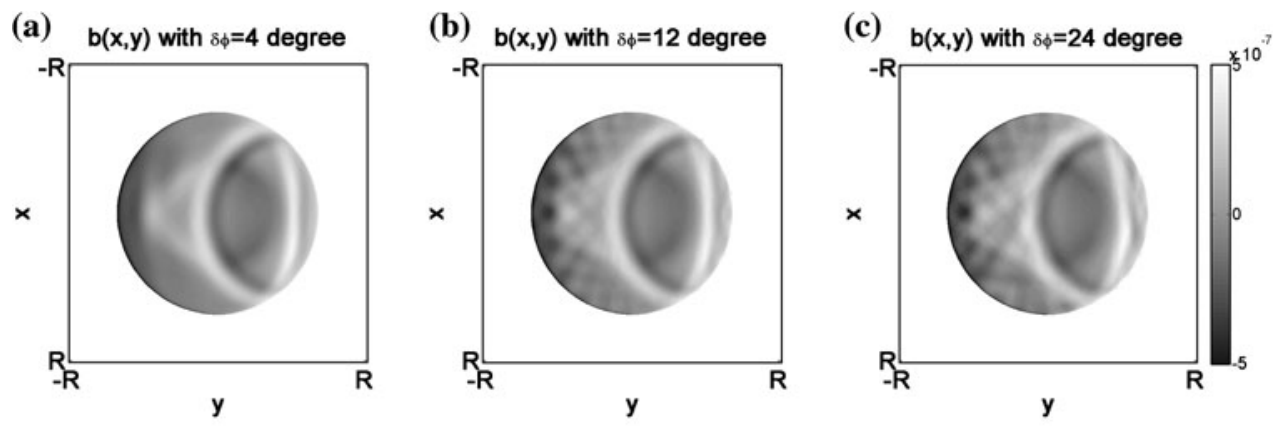

(d)
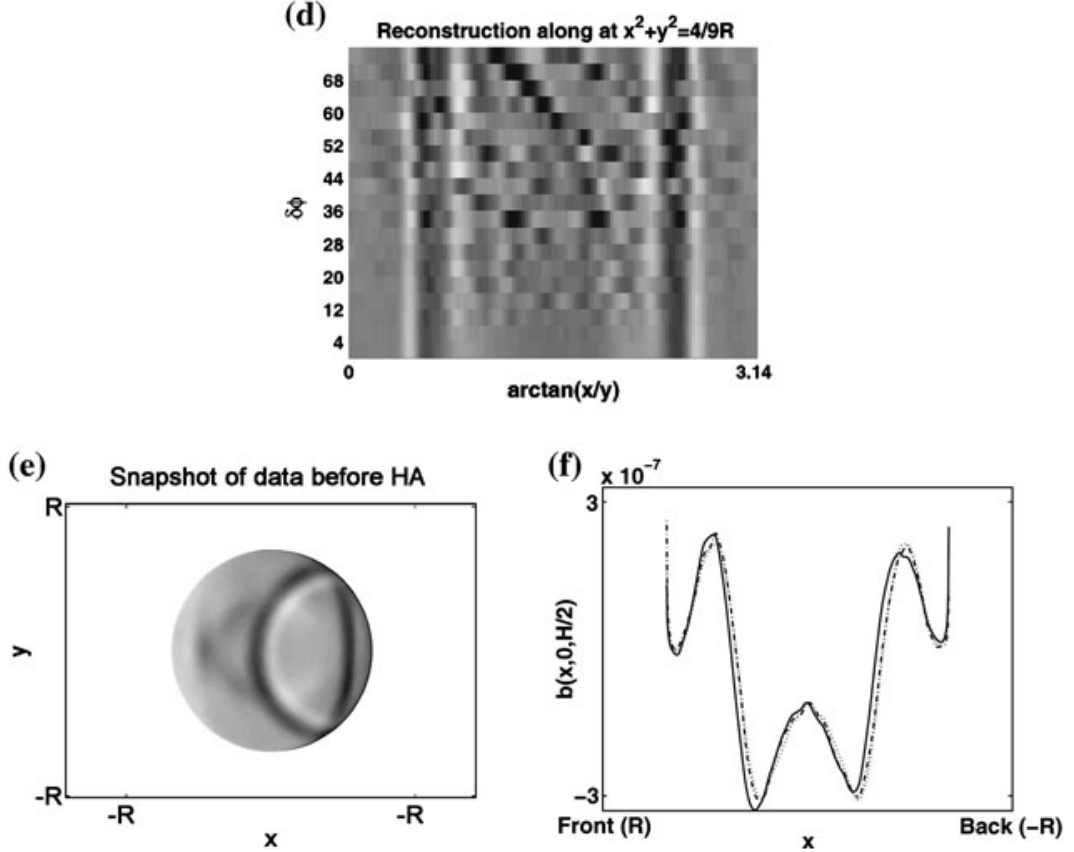

stratification was very stable and experiments prove highly repeatable, even over many hours. In an attempt to quantify the minimal angular resolution needed, we find that from $\delta \phi>36^{\circ}$ the patterns reconstructed suffer greatly from the resolution. However, it seems that even for resolutions coarser than this value the reconstruction will show key features as some angular resolutions fit the pattern better than others.

The reconstruction of the full 3D internal wave field from the oscillating sphere at the surface compares well with ray tracing the internal wave cone from a point source at the same position. At points where rays converge, the observed wave energy peaks. However, the fact that the ray tracing works well may also come as a surprise since information about amplitude and phase, and the effect of viscosity, cannot be found by means of this technique. Clearly visible in the observations are the decay of the amplitude of the internal wave cone as it radiates outwards, and phase structure discontinuities where many reflecting waves come together. However, the ray tracing does predict convergence of wave energy onto attractors, for specific forcing frequencies, at locations that in the observations also reveal strong wave motion.

In addition to considering the stationary internal wave field, the initial development of the internal wave field is accessible. Unfortunately the presentation of the evolution of $3 \mathrm{D}$ data proves very difficult to present in a $2 \mathrm{D}$ environment, and is beyond the scope of this paper. However, one may also look into the dynamics of growth and saturation of the patterns, as has been done for the $2 \mathrm{D}$ internal wave attractor (Hazewinkel et al. 2008). In recent years also tomographic PIV measurements are in development, e.g. the measurement of inertial waves by Messio et al. (2008). Eventually, the reconstructed data from synthetic schlieren should be compared with three-dimensional PIV measurements as was done for the two-dimensional case by Hazewinkel et al. (2010a).

As demonstrated by Maas (2005), the distribution of attractors is dependent on the location of the source and of the frequency. For a centered source, the attractors are 
equally spread out over all vertical planes according to the ray tracing. Even though the rays end up on attractors, their distribution ensures that they are not seen as a localized effect. In our case, we have chosen for a parameter setting where most of the attractors end up near the vertical plane that is perpendicular to the vertical plane through the source and the center. Ideally, whole parameter regimes should be scanned (by changing the source location and/or forcing frequency) to see if the ray tracing remains a reliable predictor of the internal wave patterns. However, the amount of data per experiment and long iterative procedures make it rather cumbersome to scan whole parameter regimes. Although this is merely a time- and cpu-consuming issue, it might help if optimization schemes for limited angle data sets could be implemented. The field of tomographic inversions is a very active and new methods are likely to bring new possibilities.

In conclusion, we have presented experiments in which we were able to reconstruct the $3 \mathrm{D}$ internal wave field in a paraboloidal tank with an off-centered oscillating sphere at the surface. In addition to showing the principle of tomography to work, we have demonstrated that ray tracing is a useful tool in the prediction of the internal wave patterns. In a second experiment, we confirmed that the internal wave patterns in $3 \mathrm{D}$ converge onto internal wave attractors.

Acknowledgments We thank the DAMTP-technicians for their great efforts in the experimental set-up. We thank Chrysanthi Tsimitri for her help with the experiments. J.H. is supported by a grant from the Dutch National Science Foundation N.W.O. in the Dynamics of Patterns program.

Open Access This article is distributed under the terms of the Creative Commons Attribution Noncommercial License which permits any noncommercial use, distribution, and reproduction in any medium, provided the original author(s) and source are credited.

\section{Appendix 1}

Equation 2 is derived from

$\frac{\mathrm{d}^{2} \boldsymbol{\Gamma}}{\mathrm{d} s^{2}}=\frac{\nabla_{(y, z)}}{n_{*}} n$,

where $s$ is the along view coordinate and $s=0$ at the front of the tank. We ray trace light through the refractive perturbations in the tank, $s=[0,-W]$ and to the dots pattern behind the tank, $s=[-W,-W-B]$. This means that apparent displacement vector

$\boldsymbol{\Gamma}=\int_{0}^{-B-W} \int_{0}^{s} \frac{\mathrm{d}^{2} \boldsymbol{\Gamma}}{\mathrm{d} s^{\prime 2}} \mathrm{~d} s^{\prime} \mathrm{d} s \equiv \frac{\nabla_{(y, z)}}{n_{*}} \int_{0}^{-B-W} \int_{0}^{s} n \mathrm{~d} s^{\prime} \mathrm{d} s$.

Between the tank and the dot pattern the rays are straight (no refraction; $n=0$ ), so that the integral can be split
$\boldsymbol{\Gamma}=\frac{\nabla_{(y, z)}}{n_{*}}\left[\int_{0}^{-W} \int_{0}^{s} n \mathrm{~d} s^{\prime} \mathrm{d} s+\int_{-W}^{-B-W} \int_{0}^{-W} n \mathrm{~d} s^{\prime} \mathrm{d} s\right]$.

Integrating by parts leads to

$\boldsymbol{\Gamma}=\frac{\nabla_{(y, z)}}{n_{*}}\left[\left[s \int_{0}^{s} n \mathrm{~d} s^{\prime}\right]_{0}^{-W}-\int_{0}^{-W} s^{\prime} n \mathrm{~d} s^{\prime}-B \int_{0}^{-W} n \mathrm{~d} s^{\prime}\right]$,

which, since $s=x-W / 2$, leads to

$\boldsymbol{\Gamma}=\frac{\nabla_{(y, z)}}{n_{*}}\left[\left(B+\frac{1}{2} W\right) W<n>+\int_{-W / 2}^{W / 2} x n \mathrm{~d} x^{\prime}\right]$,

Eq. 2, where $\langle n\rangle=W^{-1} \int_{-W / 2}^{W / 2} n \mathrm{~d} x$, the line of sight average of $n$.

\section{Appendix 2}

For completeness, we summarize here the 'standard' approach to tomographic reconstruction (Faris and Byer 1988). The starting point for the reconstruction is a slice considered at $z=z_{0}$ and the measured projection obtained upon integrating the deflection fields resulting from a general refraction function $f(x, y, z)$. In the new coordinates $x^{\prime}, y^{\prime}$, both functions of $\phi$, the $x^{\prime}$-axis is chosen to be parallel with the rays in that projection. This means that the projection $p\left(y^{\prime}, \phi\right)$ can be written as

$p\left(y^{\prime}, \phi\right)=\int_{-\infty}^{\infty} f\left(x^{\prime} \cos \phi-y^{\prime} \sin \phi, x^{\prime} \sin \phi+y^{\prime} \cos \phi\right) \mathrm{d} x^{\prime}$,

where the interest is in obtaining the as yet unknown function $f(x, y)$ from the observed projection $p\left(y^{\prime}, \phi\right)$. In order to find the inverse, a Fourier transform of the projection is performed

$P\left(Y^{\prime}, \phi\right)=\int_{-\infty}^{\infty} p\left(y^{\prime}, \phi\right) e^{-2 \pi Y^{\prime} y^{\prime}} d y^{\prime} \equiv \mathbb{F}_{Y^{\prime}}\left(p\left(y^{\prime}, \phi\right)\right)$,

where the capital letters denote functions and coordinates in the wavenumber domain. Substituting for $p\left(y^{\prime}, \phi\right)$, expression Eq. 13, gives

$$
\begin{aligned}
P\left(Y^{\prime}, \phi\right)= & \int_{-\infty}^{\infty} \int_{-\infty}^{\infty} f\left(x^{\prime} \cos \phi-y^{\prime} \sin \phi, x^{\prime} \sin \phi\right. \\
& \left.+y^{\prime} \cos \phi\right) \mathrm{d} x^{\prime} e^{-2 \pi \imath Y^{\prime} y^{\prime}} \mathrm{d} y^{\prime} .
\end{aligned}
$$


This expression can be rewritten in the un-rotated coordinates $x, y$ using the relations $x^{\prime}=x \cos \phi+y \sin \phi$ and $y^{\prime}=-x \sin \phi+y \cos \phi$ :

$P\left(Y^{\prime}, \phi\right)=\int_{-\infty}^{\infty} \int_{-\infty}^{\infty} f(x, y) e^{-2 \pi \imath Y^{\prime}(-x \sin \phi+y \cos \phi)} \mathrm{d} x \mathrm{~d} y$

The integral is recognized as the 2-D Fourier transform in wavenumbers $\left(k=-Y^{\prime} \sin \phi, l=Y^{\prime} \cos \phi\right)$

$P\left(Y^{\prime}, \phi\right)=\mathbb{F}_{k, l}(f(x, y))$.

Note that the left-hand side is not expressed in variables $k, l$ but in polar wavenumber coordinates $Y^{\prime}, \phi$. Nevertheless, expressing the inverse Fourier transform in coordinates $Y^{\prime}, \phi$ it is possible to find $f(x, y)$. This inverse is

$$
\begin{aligned}
f(x, y) & =\mathbb{F}_{k, l}^{-1}\left(P\left(Y^{\prime}, \phi\right)\right) \\
& =\int_{0}^{2 \pi} \int_{0}^{\infty} Y^{\prime} P\left(Y^{\prime}, \phi\right) e^{2 \pi l Y^{\prime}(-x \sin \phi+y \cos \phi)} \mathrm{d} Y^{\prime} \mathrm{d} \phi .
\end{aligned}
$$

Here $Y^{\prime}$ is the Jacobian from the Cartesian to polar wavenumber coordinate transformation. This result shows that, from the projections, the original function $f(x, y)$ can be found. With kernel $k(\eta)=\int_{0}^{\infty} Y^{\prime} e^{-2 \pi l Y^{\prime} \eta} \mathrm{d} Y^{\prime}$

and expression (14), Eq. (18) can be written as a convolution

$f(x, y)=\int_{0}^{2 \pi}[p(\eta, \phi) * k(\eta)]_{y^{\prime}(\phi)} \mathrm{d} \phi$,

where the definition of convolution $[a(\eta) * b(\eta)]_{\tau}=$ $\int_{-\infty}^{\infty} a(\eta) b(\eta-\tau) \mathrm{d} \eta$ is used. This explains the name of the algorithm, convolution back-projection. The reconstruction can be written differently when realistic measurements, with limited spatial resolution, are concerned. Then the maximum value of $Y^{\prime}$ is the Nyquist wavenumber $1 /\left(2 \Delta y^{\prime}\right)$, and a filter can be applied to cut off the higher wavenumbers.

\section{Appendix 3}

Figures 8 and 9.
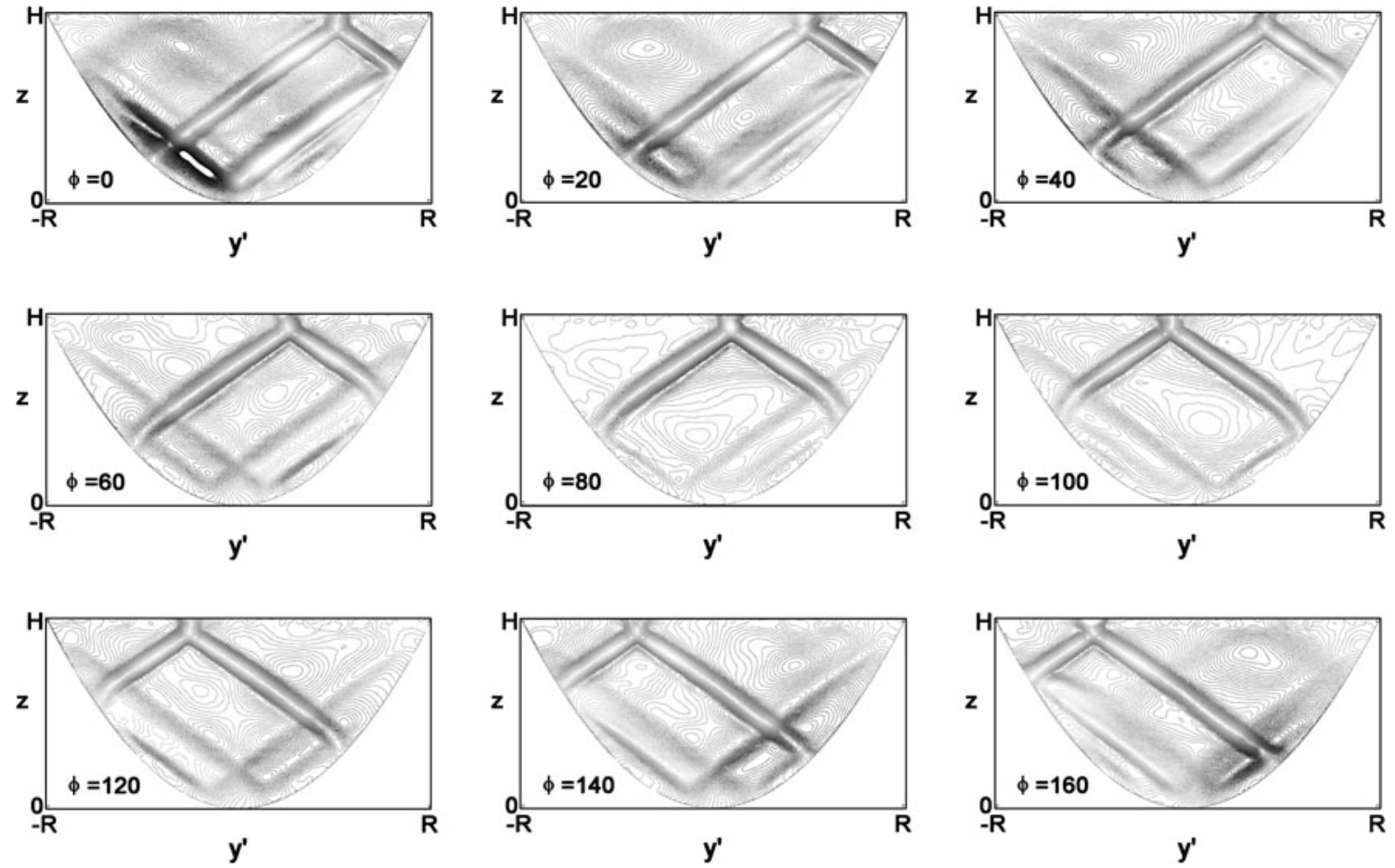

Fig. 8 Some more projections of experiment I, in addition to Fig. 2 

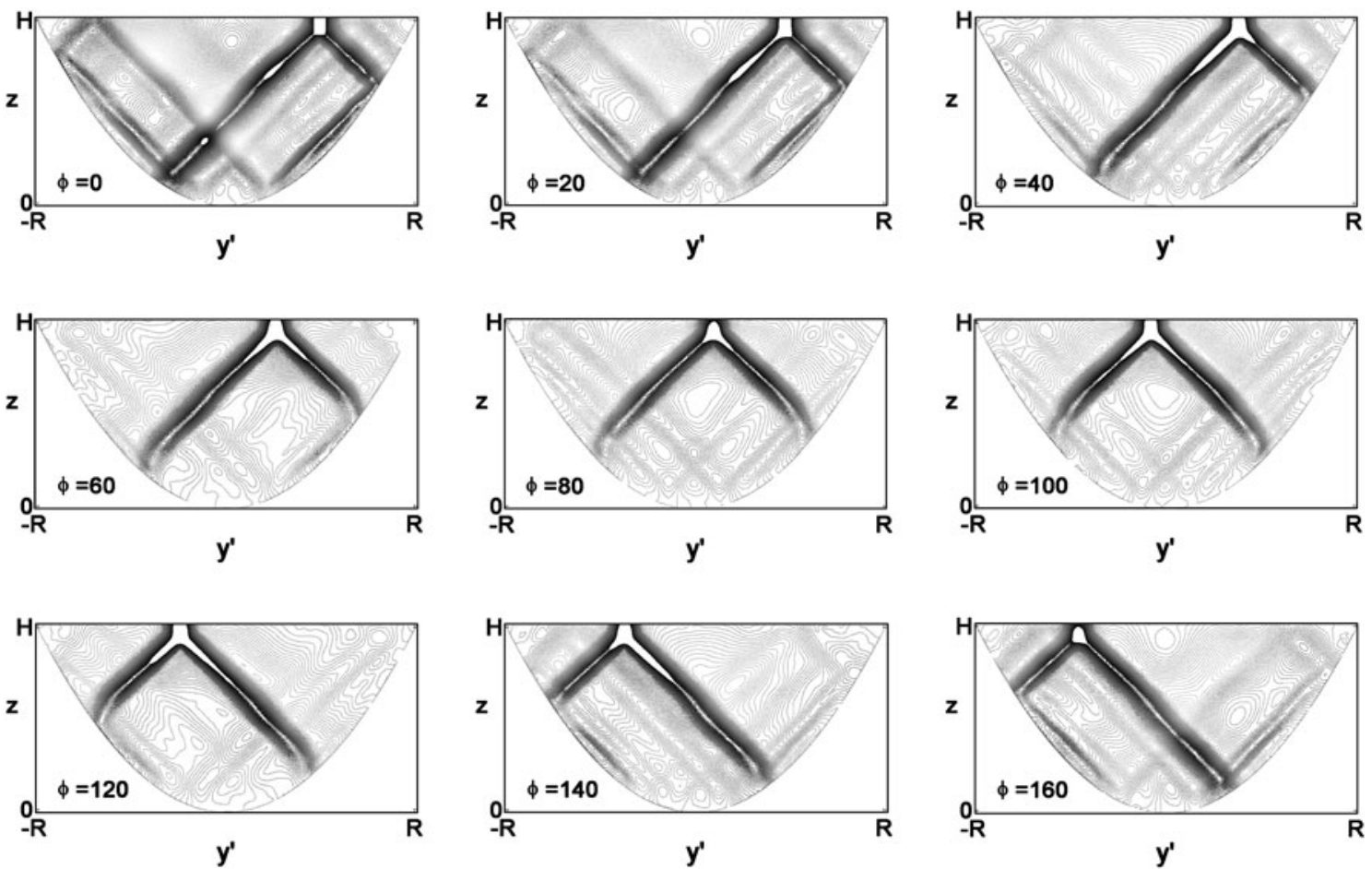

Fig. 9 Some more projections for experiment II, in addition to Fig. 5

\section{References}

Agrawal AK, Butuk NK, Gollihalli SR, Griffin D (1998) Threedimensional rainbow schlieren tomography of a temperature field in gas flows. Appl Opt 37:479-485

Baines PG (1974) The generation of internal tides over steep continental slopes. Philos Trans Royal Soc 277A:27-58

Bühler O, Muller C (2007) Instability and focusing of internal tides in the deep ocean. J Fluid Mech 588:1-28

Dalziel SB, Hughes GO, Sutherland BR (2000) Whole field density measurements by 'synthetic schlieren'. Exp Fluids 28:322-335

Dalziel SB, Carr M, Sveen KJ, Davies PA (2007) Simultaneous synthetic schlieren and PIV measurements for internal solitary waves. Meas Sci Technol 18:533-547

Dauxois T, Young WR (1999) Near critical reflection of internal waves. J Fluid Mech 390:271-295

Drijfhout S, Maas LRM (2008) Impact of channel geometry and rotation on the trapping of internal tides. J Phys Oceanogr 37:2740-2763

Echeverri P, Flynn MR, Winters CB, Peacock T (2009) Low-mode internal tide generation by topography: an experimental and numerical investigation. J Fluid Mech 636:91-108

Faris GW, Byer RL (1988) Three-dimensional beam-deflection optical tomography of a supersonic jet. Appl Opt 27:5202-5212

Goldhahn E, Seume J (2007) The background oriented schlieren technique: sensitivity, accuracy, resolution and application to a three-dimensional density field. Exp Fluids 43:241-249

Görtler H (1943) Über eine schwingungserscheinung in flüssigkeiten mit stabiler dichteschichtung. Zeitschrift für Angewandte Mathematik und Mechanik 23:65-71

Gostiaux L, Dauxois T, Didelle H, Sommeria J, Viboux S (2006) Quantitative laboratory observations of internal wave reflection on ascending slopes. Phys Fluids 18:056602
Hazewinkel J, v Breevoort P, Dalziel SB, Maas LRM (2008) Observations on the wavenumber spectrum and decay of an internal wave attractor. J Fluid Mech 598:373-382

Hazewinkel J, Grisouard N, Dalziel SB (2010a) Comparison of laboratory and numerically observed scalar fields of an internal wave attractor. Eur J Mech B/Fluids Rev

Hazewinkel J, Tsimitri C, Maas LRM, Dalziel SB (2010b) Observations on the robustness of internal wave attractors to perturbations in review

Kak AC, Slaney M (1988) Principles of computerized tomographic imaging. IEEE Inc., IEEE Press, New York

LeBlond PH, Mysak LA (1978) Waves in the Ocean. Elsevier Scientific Pub. Co., Amsterdam, p 602

Maas LRM (2005) Wave attractors: linear yet nonlinear. Int J Bifurcation Chaos 15:2757-2782

Maas LRM, Lam FPA (1995) Geometric focusing of internal waves. J Fluid Mech 300:1-41

Maas LRM, Benielli D, Sommeria J, Lam FPA (1997) Observation of an internal wave attractor in a confined stably-stratified fluid. Nature 388:557-561

Mercier MJ, Garnier NB, Dauxois T (2008) Reflection and diffraction of internal waves analyzed with the hilbert transform. Phys Fluids 20:086,601

Messio L, Morize C, Rabaud M, Moisy F (2008) Experimental observation using particle image velocimetry of inertial waves in a rotating fluid. Exp Fluids 44:519-528

Mowbray DE, Rarity BSH (1967) A theoretical and experimental investigation of the phase configuration of internal waves of small amplitude in a density stratified liquid. J Fluid Mech 28:1-16

Onu K, Flynn MR, Sutherland BR (2003) Schlieren measurement of axisymmetric internal wave amplitudes. Exp Fluids 35:24-31

Richard H, Raffel M (2001) Principle and applications of the background oriented schlieren (bos) method. Meas Sci Technol 12:1576-1585 
Scase MM, Dalziel SB (2006) Internal wave fields generated by a translating body in a stratified fluid: an experimental comparison. J Fluid Mech 564:305-331

Srivastava A, Muralidhar K, Panigrahi PK (2005) Reconstruction of the concentration field around a growing kdp crystal with schlieren tomography. Appl Opt 44:5381-5392

Sutherland BR, Hughes GO, Dalziel SB, Linden PF (1999) Visualisation and measurement of internal gravity waves by 'synthetic schlieren', part I: vertically oscillating cylinder. J Fluid Mech 390:93-126
Tabaei A, Akylas TR (2003) Nonlinear internal gravity wave beams. J Fluid Mech 482:141-161

Thomas NH, Stevenson TN (1972) A similarity solution for viscous internal waves. J Fluid Mech 54(3):495-506

Weast RC (1981) Handbook of chemistry and physics. CRC Press, Boca Raton

Yick K, Stocker R, Peacock T (2006) Microscale synthetic schlieren. Exp Fluids 42:41-48 